\title{
Resident competencies and work hours: are they mutually exclusive
}

- or many years significant changes - in residency education in the US have occurred primarily in the required curriculum for each specific training discipline. Now, residency training is undergoing dramatic revision across all graduate medical education programmes as part of the General Requirements for Graduate Medical Education. Two issues are driving this change-the assessment of resident education based on a set of general competencies, and a "legislated" reduction in work hours.

In the US, residency training is under the direction of the Accreditation Council for Graduate Medical Education (ACGME). ACGME establishes the core training requirements for all accredited postgraduate training programmes. These core requirements are clearly articulated and include the requirements for sponsoring institutions. Within its mandate for establishing these core requirements, ACGME has developed two major revisions that must now be incorporated and instituted by all programmes in order to remain accredited.

In February 1999, ACGME announced its recommendations for outcome assessment of trainees. This is highlighted by the ACGME requirement that all accredited programmes must measure resident competency in the following areas:

- Patient care that is compassionate, appropriate, and effective for the treatment of health problems and the promotion of health.

- Medical knowledge about established and evolving biomedical, clinical, and cognate sciences and the application of this knowledge to patient care.

- Practice based learning and improvement that involves investigation and evaluation of their own patient care, appraisal and assimilation of scientific evidence, and improvements in patient care.

- Interpersonal and communication skills that result in effective information exchange and teaming with patients, their families, and other health professionals.

- Professionalism, as manifested through a commitment to carrying out professional responsibilities, adherence to ethical principles, and sensitivity to a diverse patient population.
- Systems based practice, as manifest by actions that demonstrate an awareness of and responsiveness to the larger context and system of health care and the ability to effectively call on system resources to provide care that is of optimal value.

In addition to endorsing these competencies, ACGME is also requiring training programmes to modify the process for resident evaluation and move towards more objective measures of competency and combine this with a system that demonstrates improved performance over the course of the training programme. Specific objectives for residents to demonstrate scholarly progress in several of the competencies must now be incorporated into each training programme. Finally, programmes are expected to define and develop the skills, knowledge, and attitudes that are required for residents to achieve the level of a new practitioner in their respective field.

The educational experiences that residents must complete to achieve the General Competencies remain under the direction of the Residency Review Committee (RRC) for each discipline. In the past, each Residency Review Committee has been very specific in defining the educational experiences (inpatient versus ambulatory, intensive care versus general wards, etc) that needs to be included in each accredited training programme. By endorsing these competencies and utilising an approach that stresses educational outcomes, ACGME may provide more flexibility for each RRC as they establish their specific training requirements.

With respect to work hours for residents, finally, the US is moving towards the standards established by most of our European colleagues. The Board of Directors of ACGME, in response to public perception that long work hours impact on patient safety and some new data about the effect of fatigue on performance, have recommended the following guidelines for resident work hours:

- Residents must not work for more than 80 hours per week, averaged over a four week period. If residents are in the hospital, even if they are sleeping, this is counted as part of the 80 hour week. Individual programmes may apply to their sponsoring institutions to increase this limit up to 88 hours per week. Programmes must provide educational rationale to support this increase.

- Residents must have one full day out of seven free of patient care duties.

- In-house call may not occur more than every third night averaged over a four week period.

- Continuous time on duty is limited to 24 hours. An additional six hours of time may be used for care for current patients, transfer of care, or educational activities. No new patient care may occur during this six hour time period, with the exception of care provided in the residents continuity clinic.

- Residents are required to have a minimum of 10 hours off between duty periods.

These work hour requirements are consistent with those that exist in many European nations.

Are residency competencies and work restrictions compatible? Can we train residents in the same length of time that we have in the past, despite the new restrictions? Certainly the growth of medical knowledge, specifically new diagnostic tests and novel therapies, will make this difficult. However, this may be a moot question, because virtually no one is considering extending residency beyond the traditional three years in the US for paediatricians, internists, and family practitioners. Residents and hospitals would probably not tolerate any extension. Could additional residents be hired? Again, this is unlikely to occur for numerous reasons, including financial limitations and continuing issues about the number of physicians in the US.

The more appropriate question is what new educational strategies are needed to meet the ACGME competency requirements and mandate of a shorter work week. First and foremost, each programme must now review their educational programme and provide the appropriate balance between service and educational experiences. Within the defined work hour requirements, each programme must be sure that the experiences required of trainees meet an educational goal defined by the leadership of the programme and consistent with the ACGME competencies. Service driven rotations may need to be 
reviewed and/or eliminated. Second, technology will need to assist us in meeting our training goals. Computers, hand held devices, and simulation devices, must be incorporated into training, so that residents can be exposed to more critical issues in less time. Third, we must teach residents to become life-long learners. They must learn how to incorporate advances in medicine and a commitment to quality improvement with patient centred care throughout their entire career. As physicians we continue to learn for many years beyond our training programme. Finally, the reduction in work hours will increase the number of times patients are "handed off" to a fellow resident. Technology will be necessary to enhance the transfer of information and to ensure patient safety.
Advances have come slowly to resident education. The dramatic reduction in length of stay for patients, and the amount of diagnostic and therapeutic testing that is currently performed, makes life for residents far more complex than 20 years ago.

R Vinci

Boston University School of Medicine, Boston Medical Center, Boston, MA, USA; bob.vinci@bmc.org

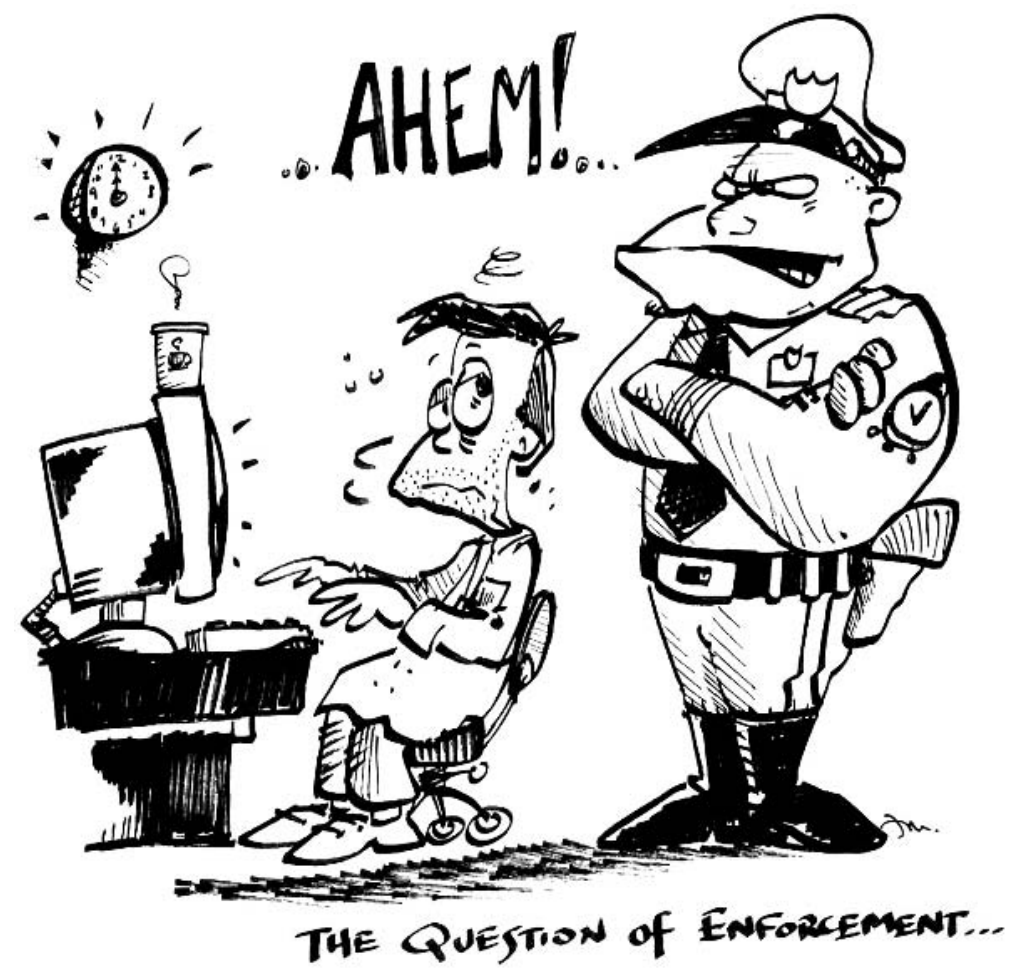

Illustration by Jack Maypole, MD 